\title{
KINEMATICS AND DYNAMICS ANALYSIS OF EXOSKELETON TRAINING ROBOT BASED ON THE HUMAN BIOMECHANICAL MODEL
}

\author{
Yang Zhao \\ Department of Physical Education, Shaanxi International Business College, Xi'an, Shaanxi Province, \\ 712046, China \\ E-mail: zhaoyang12@yahoo.com
}

\begin{abstract}
Exoskeleton robot is a recently emerged human and electromechanics integrated technology which perfectly combines human wisdom with mechanical strength and has good application values and market prospects. In order to better achieve human-computer interaction, optimize the performance of the exoskeleton training robot, reduce muscle burden and enhance human strength and endurance, this study investigated the exoskeleton training robot based on the human biomechanical model after fully understanding the theories of ergonomics, kinematics and dynamics. The robot design fully considered the human biomechanical parameters and realized the adjustment on the mechanical and control system by setting structural limits based on the degree of freedom of the joints of the human body. Besides, the feasibility and follow - up function of the exoskeleton robot were verified by testing. The kinematics and dynamics analysis of the exoskeleton training robot based on the human biomechanical model can help better control and optimize the design structure of the robot, enhance the power-assisted effect, and help the users to enhance their strength and physical fitness and carry out more scientific and safe training.
\end{abstract}

Keywords: Path tracking, Kinematics model, GNSS receiver, Autonomous Vehicle.

\section{Introduction}

The exoskeleton robot [1] is a wearable mechanical device with protection and assistance functions. It uses the computer to establish the calculation model of the human body and the machine, incorporates the physiological characteristics of the human body, and simulates the various actions of humans operating machines, and it has been widely used in military and medical rehabilitation fields. The exoskeleton helps people run faster and jump higher. With the development of science and technology, many experts and scholars have carried out relevant studies. Bechet $\mathrm{F}$ et al. [2] believed that exoskeletons were external structural mechanisms designed to be mounted on human body so as to support or enhance its physical abilities.

Margaritescu M et al. [3] developed a robot structure with high preciseness, high rigidness and fast response using LabVIEW modelling and control method based on hybrid kinematics. Xing K et al. [4] explored the key techniques and research significance of the current design of external skeletal robots. In this study, an external skeletal training robot model based on human biomechanics was constructed, and the kinematics and dynamics analysis were used to optimize its structure, which makes the designed robot more safe and practical to meet the needs of training for users.

\section{Exoskeleton robot}

Exoskeleton robot technology can provide human body with a wearable mechanical structure which integrates sensing, control, information and mobile computing [5]. It can help human fulfil actions which cannot be completed independently previously in complex environment. It is usually applied in the fields of military and medicine.

\subsection{Application of exoskeleton robot in military field}

Many countries have designed an exoskeleton robot to improve the single combatant ability. Wearing exoskeleton robots, soldiers can use multiple equipments, have stronger single combatant ability and move fast in more poor environments without consuming much physical power. Exoskeleton robots can produce a huge effect on improving single combatant ability. 


\subsection{Application of exoskeleton robot in medical field}

In medical field, exoskeleton robot is a kind of novel wearable light-weight robot. It can provide assistance using drivers and help the disabled recover walking ability and patients fulfil actions which cannot be completed independently. Moreover it can avoid pressure sores which are induced by long-time sitting on wheelchair, improve the heart health condition of patients, and exercise muscle strength.

\section{Design of exoskeleton training robot}

\subsection{Overall design of the of exoskeleton training robot}

The exoskeleton training robot designed in this study is mainly used for human lower limb exercise training to enhance users' physical strength. During the design process, the human biomechanical model [6] was fully considered. The mechanism movement of the lower extremity exoskeleton includes three rotary movement of degree of freedom of the hip joint [7], one rotary movement of degree of freedom of the knee joint [8] and two rotary movement of degree of freedom of the ankle joint [9]. The setting of the mechanical limit depends on the range of degree of freedom of the joints of the body [10]. As shown in figure 1 , the mechanical structure of the exoskeleton training robot mainly includes the thigh connecting rod, the calf connecting rod, the leggings, the foot plate, etc. Redundant degree of freedom [11] was adopted structurally to enhance flexibility [12]. The I - beam half - enclosed design was applied, with the thighs and calves connected with leggings and each component made of 7075 aluminum alloy with close structure and high strength. The muff-coupling way makes it possible to adjust the length of the connecting rods to meet the needs of users of different height. The thigh and the calf were equipped with an acceleration sensor and a pressure sensor, and a thin film pressure sensor is mounted on the foot [13]. The motor driver is mounted in the structural cavity near the joint and the design of an elastic series element is added at each joint.

The control system is composed of the main controller, the node controller and the host computer $\mathrm{PC}$, to realize the precise control of the joint movement, the movement adjustment of the movement training and the simple human-computer interaction control.

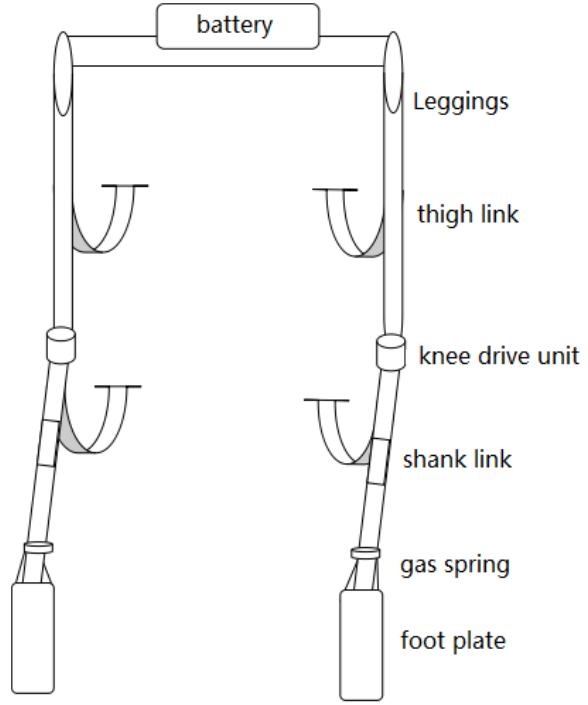

Figure 1: Schematic diagram of simplified design of an exoskeleton robot

\subsection{Hardware design of the exoskeleton robot}

\subsubsection{Design of sensors}

The sensors used in this study included acceleration sensors, pressure sensors, EMG sensors and photoelectric encoders. The acceleration sensor and pressure sensor were installed at the thigh and calf to obtain the real-time motion parameters of each joint; a pressure sensor was installed to the foot to determine the movement speed and direction according to pressure changes; angular velocity sensors were distributed at the rotation part of each joint. During the training, the exoskeleton robot detected the user's motion through the sensors and drove the movement of the lower limbs relying on the movement information stored in the processor.

\subsubsection{Controller design}

The main controller, node controller and PC together form the control system of the exoskeleton training robot. The STM32F4 high-performance microcontroller is selected as the primary controller. It integrates the new DSP and FPU instructions, and its $168 \mathrm{MHz}$ high-speed performance enhances the control algorithm execution speed and code

efficiency, realizes the transmission of the data collected by the sensors from the electromechanical controller to the main controller and bears a lot of control algorithm operations and data transmission tasks. The communication interfaces used are CAN port, serial port and network port and the former two realizes the TTL level conversion. 
The TJA1050 is used to provide differential transmission performance for the bus and differential receiver performance for the CAN port. Circuits include crystal oscillator circuit, reset circuit and filter circuit. STM32F107 was selected for node control, which integrates a variety of highperformance industrial standard interfaces. Besides, different models of STM32 products share perfect compatibility in the pin and software, can achieve the control of the external skeleton robot joints and send signals collected by sensors to the main controller. The control system workflow is shown in Figure 2. The main controller processes the data transmitted by the node controller, such as the joint angles of exoskeleton, and sends drive commands to the actuator body model and motor. After the expected rate is reached through torque control, the control signal feeds the data information back to the main controller and the main controller communicates with the PC through the network port so as to achieve the training data recording and training mode settings.

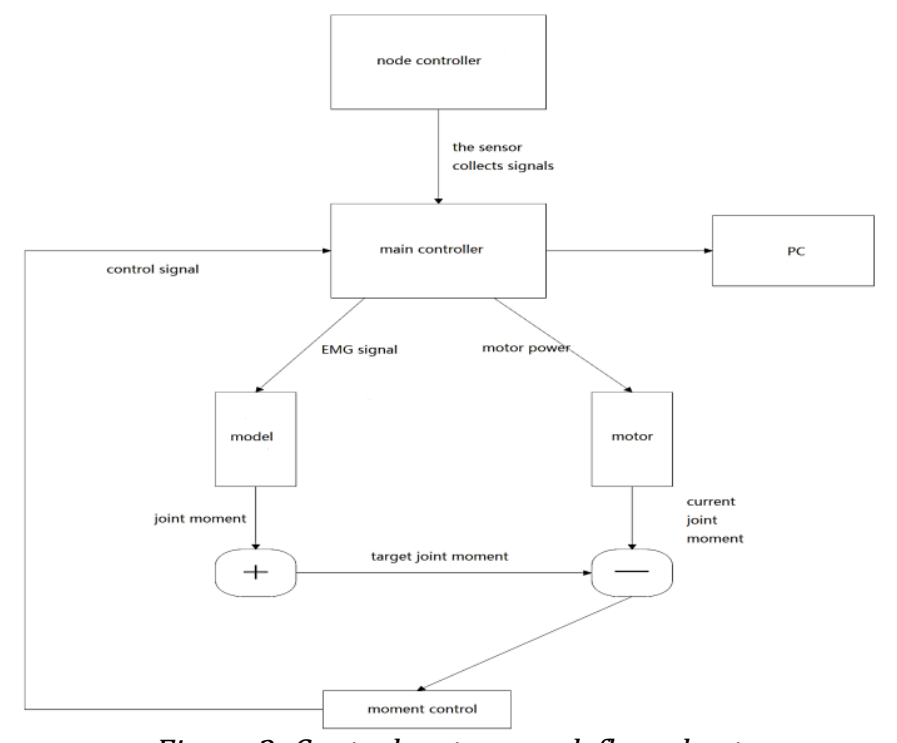

Figure 2: Control system work flow chart

\subsubsection{Design of the driver}

In this design, the motor drive mode with simple driven power, high speed and position accuracy and good speed control capacity was selected, which can respond quickly to the controller's instructions and quickly complete the required action. Hence, the Elmo driver with simple and efficient multi-axis control technology and low EMI is chosen to support incremental encoders, provide feedbacks of the exoskeleton training robot movement information to the main controller through the encoders, control the speed and location and realize the closed-loop current control through the internal current feedback. Besides, power supply to the drive motor and the control system is provided by batteries.

\subsection{Design and analysis of the exoskeleton robot}

\subsubsection{Kinematics analysis}

External skeletal robot kinematics [14] is divided into positive kinematics and inverse kinematics. Positive kinematics checks whether the robot movement meets the target requirements, and the inverse kinematics tests whether the end of the robot rod can reach the desired position. The D-H method [15] is used to complete the external skeleton robot modeling and perform kinematics analysis. Since the lower limb exoskeleton robot is the same in mechanical structure and movement mode, the model can be simplified as a two-link model, as shown in figure 3 .

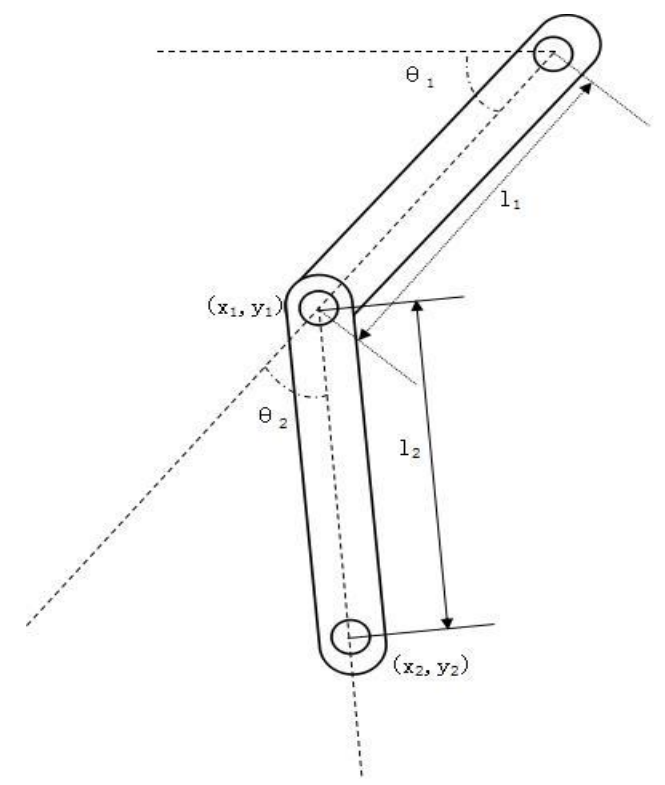

Figure 3: Lower limb exoskeleton robot two-link model 
The D-H matrix of the rotating joint was represented by the following formula, where $\theta_{n}$ refers to the included angle between rod piece $n$ and $\mathrm{n}+1,{ }^{l}$ refers to the length of rod piece n, ${ }^{\alpha}{ }_{n}$ refers to the rotated angle of rod piece $\mathrm{n}$ and $b_{n}$ refers to the offset length between rod piece $n$ and $n+1$.

$$
T_{n}^{n-1}=\left[\begin{array}{cccc}
\cos \theta_{n} & -\cos \alpha_{n} \sin \theta_{n} & \sin \alpha_{n} \sin \theta_{n} & l_{n} \cos \theta_{n} \\
\sin \theta_{n} & \cos \alpha_{n} \cos \theta_{n} & -\sin \alpha_{n} \cos \theta_{n} & l_{n} \sin \theta_{n} \\
0 & \sin \alpha_{n} & \cos \alpha_{n} & b_{n} \\
0 & 0 & 0 & 1
\end{array}\right]
$$

The D-H matrix of the moving joint was represented by the following formula:

$$
T_{n}^{n-1}=\left[\begin{array}{cccc}
\cos \theta_{n} & -\cos \alpha_{n} \sin \theta_{n} & \sin \alpha_{n} \sin \theta_{n} & 0 \\
\sin \theta_{n} & \cos \alpha_{n} \cos \theta_{n} & -\sin \alpha_{n} \cos \theta_{n} & 0 \\
0 & \sin \alpha_{n} & \cos \alpha_{n} & b_{n} \\
0 & 0 & 0 & 1
\end{array}\right]
$$

Substitute the robot model link into the matrix, then the following was obtained:

$$
T_{2}^{0}=\left[\begin{array}{cccc}
\cos \left(\theta_{1}+\theta_{2}\right) & -\sin \left(\theta_{1}+\theta_{2}\right) & 0 & l_{1} \cos \theta_{1}+l_{2} \cos \left(\theta_{1}+\theta_{2}\right) \\
\sin \left(\theta_{1}+\theta_{2}\right) & \cos \left(\theta_{1}+\theta_{2}\right) & 0 & l_{1} \sin \theta_{1}+l_{2} \sin \left(\theta_{1}+\theta_{2}\right) \\
0 & 0 & 1 & 0 \\
0 & 0 & 0 & 1
\end{array}\right]
$$

Thus, the kinematic relationship of the exoskeleton training robot was obtained:

$$
\begin{aligned}
& x_{1}=l_{1} \cos \theta_{1}, y_{1}=l_{1} \sin \theta_{1}, \\
& x_{2}=l_{1} \cos \theta_{1}+l_{2} \cos \left(\theta_{1}+\theta_{2}\right) \text { and } \\
& y_{2}=l_{1} \sin \theta_{1}+l_{2} \sin \left(\theta_{1}+\theta_{2}\right) .
\end{aligned}
$$

By substituting the angle range of each joint of the lower limbs into the above-mentioned kinematic model, a cloud map of the movement range of the exoskeleton robot was obtained. Based on the mechanical structure of the robot body structure and the actual position, further solution was searched for. The relative coordinate system was applied to the structural adjustment of the exoskeleton robot, which makes the exoskeleton robot meet the requirements of sports training.

\subsubsection{Dynamics analysis}

During walking, the load of joints of the legs becomes the highest when the feet land. Therefore, it was taken for modelling analysis. The motion state of the robot was regarded as single-leg support. Therefore, the two legs of the robot were paralleled and symmetrical. In this study, single leg was selected for study. Model was established by regarding the motion state of robots as single-leg support, as shown in figure 4.

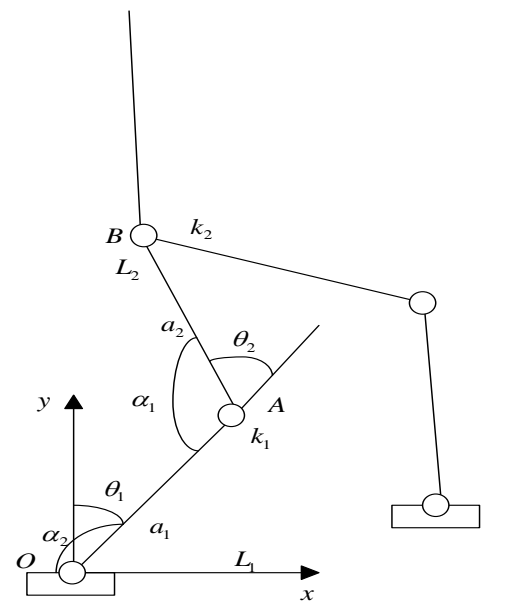

Figure 4: the simplified model of single-leg support

In the kinetic analysis of the exoskeleton robot, Lagrangian equation [16] was used. The following equation was obtained according to fig. 3.

$$
M(\theta) \ddot{\theta}+C(\theta, \dot{\theta}) \dot{\theta}+P(\theta)=H+l
$$

$$
\theta=\left[\theta_{1}, \theta_{2}, \theta_{3} \ldots \theta_{n}\right]^{H}, H=\left[0, H_{1}, H_{2}, H_{3} \ldots H_{n}\right]^{H}
$$

$\boldsymbol{M ( \boldsymbol { \theta } )}$ was a $5 * 5$ inertia matrix, $\boldsymbol{P}(\boldsymbol{\theta})$ was a $5 * 1$ gravity matrix, $C(\theta, \dot{\theta})$ was a matrix with regard to centrifugal force and Coriolis, $\boldsymbol{H}$ was a $5 * 1$ driving force moment vector, and $\boldsymbol{l}$ was the moment of force exerted on robots by users.

The following equation was obtained through calculation. 


$$
P_{m n}=\left\{\begin{array}{c}
I_{m}+k_{m} l_{m}{ }^{2}+a_{m}\left(\sum_{n=m+1}^{5} k n\right) h_{n}{ }^{2} \quad n=m \\
a_{m} k_{n} l_{n} h_{m}+a_{m} a_{n}\left(\sum_{s=m+1}^{5} k_{s}\right) h_{m} h_{n} \quad n>m \\
P_{m n} n<m
\end{array}\right.
$$$$
r_{m}=k_{m} l_{m} g+a_{m}\left(\sum_{n=m+1}^{5} k_{n}\right) h_{m} g
$$

Where $I$ stands for inertia tensor and $h$ stands for the distance of adjacent connecting rods.

\section{Test of the exoskeleton training robot}

In order to verify the feasibility and function of the exoskeleton training robot, relevant testing was carried out. The objects of this test were two men athletes from the Shaanxi Athletics Association who had similar height, weight and training periods.

\subsection{Feasibility test of the exoskeleton robot}

Taking the walking race exercise as an example, the exoskeleton robot could assist the lower limb movement in the process of human walking, and enhance the function of the lower limbs, but the premise was that the gait of the exoskeleton robot and a normal person were basically the same. In this test, the two athletes were asked to wear exoskeleton training robots which would then drive them to move. Then, the MATLAB software was applied to make a comparison between the motor trajectory of each joint in the robot training process and their actual trajectory in the process of normal sports training.

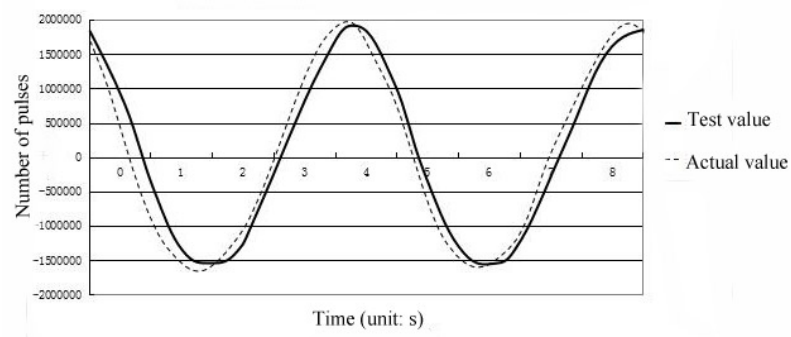

Figure 5: Comparison of the motor tracking curve of the hip joint with their actual trajectory

As shown in Figure 5, the experimental trajectory of the hip joint coincided with its actual trajectory, and the gait law of the exoskeleton robot was consistent with the gait law in normal exercise, suggesting that the exoskeleton training robot was feasible as it could effectively drive the user to carry out sports training and make proper gait adjustment adapting to different movement needs.

\subsection{Functional test of the exoskeleton robot}

In order to test the follow-up function and the assisting situation of the exoskeleton robot, a single joint following motion experiment was carried out.

The two athletes were asked to wear the exoskeleton training robot and their thighs and calves were ensured to be fixed with the robot through the leggings. By keeping still of the thighs of the subjects and making cyclic swing of their calves, the experiment was carried out. At this time, the knee motor was in the servo state and the hip motor was still fixed. Because the pressure sensor was installed at the bundling device, when the athlete's leg swung, the pressure change occurred at the binding device, which causes the resistance of the pressure sensor to change. Then, the node controller transmitted the collected sensor information to the main controller, and the main controller controlled the motor to produce the corresponding movement.

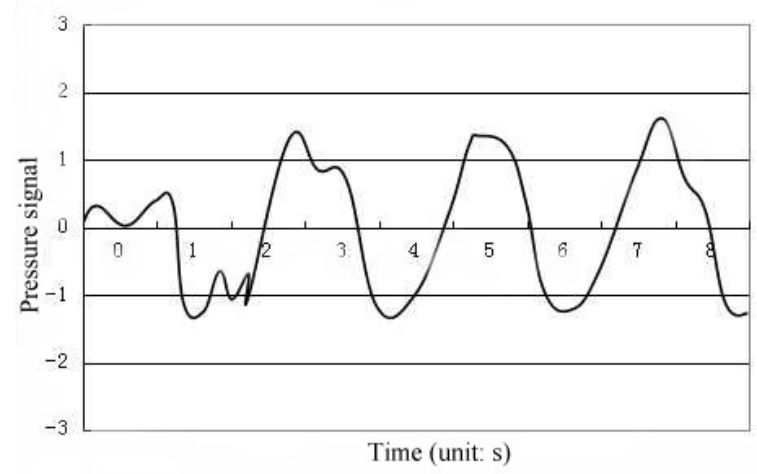

Figure 6: Pressure sensor measured pressure signal situation

As shown in Figure 6, the amplitude of the pressure signal measured by the pressure sensor was small and the signal had obvious periodicity. It could be noted that the resistance between the human and the exoskeleton training robot was small.

When the athlete swung back and forth their calves, good synchronous movement could be realized due to the negligible resistance, with good following effect, which helped improve the training effect and enhance the athlete's physical strength.

\subsection{Biomechanical test of the exoskeleton robot}

As shown in figure 7, when motion started, the length of location of mass center of both the right 
thign and crus was about $100 \mathrm{~mm}$; with the changes of motion, the length of location of mass center of the right thign fluctuated between $100 \mathrm{~mm}$ and $-100 \mathrm{~mm}$ and that of the left thign fluctuated between $100 \mathrm{~mm}$ and $200 \mathrm{~mm}$; the length of location of mass center of the left thign fluctuated between $150 \mathrm{~mm}$ and 200 $\mathrm{mm}$; the length of location of mass center of the left crus fluctuated between $150 \mathrm{~mm}$ and $0 \mathrm{~mm}$. It could be noted that the track of location of mass center of the right thign was similar to that of the left crus, suggesting the motion state of the right thign and the left crus was similar and the motion state of the left thign and right crus was similar as well. It was concluded that the mass center was stable when the exoskeleton robot moved, which reduced energy consumption and conformed to the motion characteristics of human body. The initial moment of force of the hip joint was 2800 Newton-mm and fluctuated between 5000 Newton-mm and 1500 Newton-mm with the changes of motion. The initial moment of force of the knee joint was 4900 Newton$\mathrm{mm}$ and fluctuated between 6000 Newton-mm and 1500 Newton-mm with the changes of motion. The moment of force of the hip and knee joints was highly similar. The curves of moment of force of the two joints were obtained through simulation tests.

The simulation results could be regarded as the design basis for the control system of robots, which can provide an evidence for the development of exoskeleton robot.
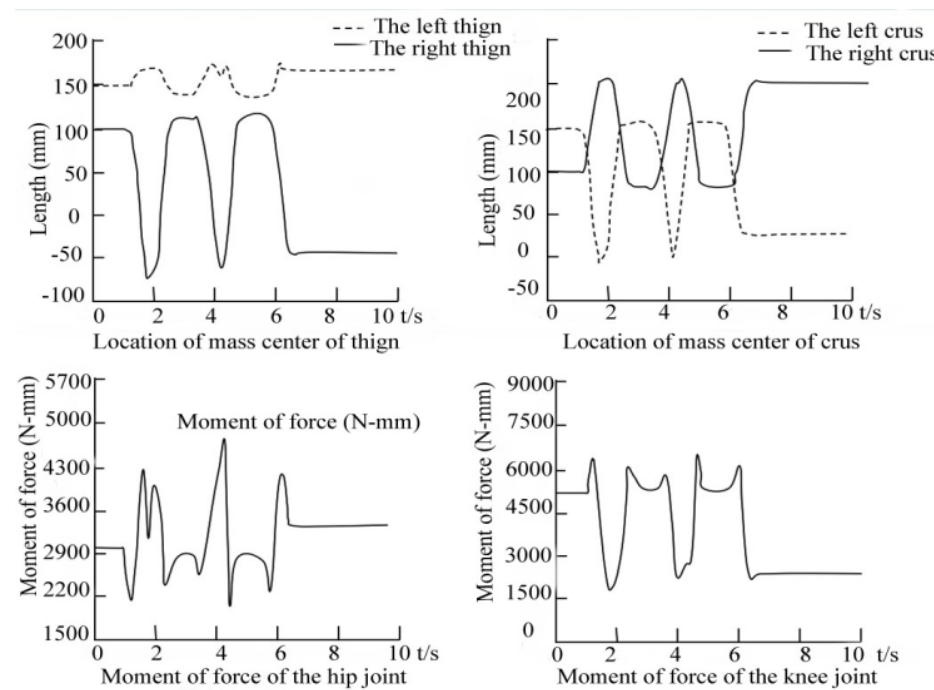

Figure 7: The kinematics and dynamics simulation tests on the exoskeleton robot

\subsection{Optimization test of the exoskeleton robot}

At the present stage, the structural properties of exoskeleton robots still have some optimization space. A simulation experiment was carried out by introducing the 3D model of the exoskeleton robot in the ADAMS software [17], which validated the results obtained by the kinematics and dynamics modeling analysis to provide a basis for the subsequent optimization design. The testing result showed that the changes of displacement velocity and angular velocity of the knee joint of the exoskeleton robot were not obvious in the leg support phase but very obvious when the feet touched the ground which produced a strong impact.

Therefore, the speed of the driver needs to be adjusted. During the lower limb movement, the ankle joint will bear a constantly changing support reverse force due to the interference of the ground, which puts forward some demands on the cushioning device.

\section{Conclusion}

The designed exoskeleton training robot which is based on the human biomechanical model combines ergonomics and mechanical and electrical integration theories and can help enhance the user's strength and endurance as well as improve their training effect. Through the kinematics and dynamics modeling analysis, the information such as moment, displacement and energy consumption of each joint was obtained, which provides a theoretical basis for the optimization of the structure, making the design of the exoskeleton robot more scientific and reasonable. Nevertheless, this design is still at the structural level and the control and electrical parts are yet to be further studied. In conclusion, kinematics and dynamics analysis on the human biomechanical model based exoskeleton training robot is beneficial to the optimization of the structure and performance of the robot, the enhancement of assistance effect and the training of trainers. 


\section{References}

[1] Li, N., Yan, L., Qian, H., Wu, H., Wu, J. and Men, S., Review on lower extremity exoskeleton robot, Open Automation \& Control Systems Journal, 2015, 7(1):441-453.

[2] Bechet, F. and Ohnishi, K., Electro-hydraulic force transmission for rehabilitation exoskeleton robot IEEE, International Workshop on Advanced Motion Control. IEEE, 2014:260-265.

[3] Li Z., sEMG-based joint force control for an upperlimb power-assist exoskeleton robot, IEEE Journal of Biomedical \& Health Informatics, 2014, 18(3):1043-1050.

[4] Xing, K., Zhao, X. H. and Chen, W., Research status and development trend of exoskeleton robot, Medical Equipment, 2015, 36 (1): 104-107.

[5] Park, Y. L., Chen, B. R., Pérezarancibia, N. O. Young, D., Stirling, L., Wood, R. J., Goldfield, E. C. and Nagpal, R., Design and control of a bioinspired soft wearable robotic device for anklefoot rehabilitation, Bioinspiration \& Biomimetics, 2014, 9(1):016007.

[6] Carmichael M G, Liu D K., Human Biomechanical Model Based Optimal Design of Assistive Shoulder Exoskeleton, Springer Tracts in Advanced Robotics, 2015, 105:245-258.

[7] Sangeux, M., Pillet, H. and Skalli, W., Which method of hip joint centre localisation should be used in gait analysis?, Gait \& Posture, 2014, 40(1):20-25.

[8] Han, Y. L., Xu, Y. X., Gao, H. T., Zhu S. Q. and Shi Y., Study on exoskeleton swing control of knee joint based on admittance control, Acta Automatica Sinica, 2016, 42 (12).

[9] Sakaguchi, M., Ogawa, H., Shimizu, N., Kanehisa H, Yanai, T. and Kawakami Y., Gender differences in hip and ankle joint kinematics on knee abduction during running, European Journal of Sport Science, 2014, 14(sup1):S302-S309.
[10] Xu, H., Bloswick, D. and Merryweather, A., An improved OpenSim gait model with multiple degrees of freedom knee joint and knee ligaments, Computer Methods in Biomechanics \& Biomedical Engineering, 2015, 18(11):1217.

[11] Abadi, B. N. R., Taghvaei, S. and Vatankhah, R., Optimal Motion Planning of a Planar Parallel Manipulator with Kinematically Redundant Degrees of Freedom, Transactions-Canadian Society for Mechanical Engineering, 2016, 40(3):383-397.

[12] Gunasekara, J. M. P., Gopura, R. A. R. C., Jayawardena, T. S. S., Redundant upper limb exoskeleton robot with passive compliance, International Conference on Information and Automation for Sustainability. IEEE, 2015:1-6.

[13] Kazaryan, A. A. and Strel'Tsov, E. V., A Thin-Film Capacitance Pressure Sensor, Measurement Techniques, 2015, 57(12):1403-1410.

[14] Fekrache, D. and Guiatni, M., Kinematics and design of a 5 DoF exoskeleton for the rehabilitation of the upper limb, International Conference on Electrical Engineering. IEEE, 2015:1-5.

[15] Wen, S., Ma, Z., Wen, S., Zhao, Y. and Yao, J., The study of NAO robot arm based on direct kinematics by using D-H method, Control (CONTROL), 2014 UKACC International Conference on. IEEE, 2014:515-518.

[16] Rubinstein, Y. A. and Solomon, J. P., The degenerate special Lagrangian equation, Advances in Mathematics, 2017, 310:889-939.

[17] Salah, W., Mohamed,_F., Abdel-Rahman, A. K. and Rassoul, S. A., Dynamic Simulation and Optimization of Rhombic Drive Stirling Engine Using MSC ADAMS Software, Procedia Engineering, 2016, 22:754-761. 